\title{
La regulación de los datos abiertos
}

The regulation of open data

\author{
Fernando GALINDO AYUDA \\ Facultad de Derecho, Universidad de Zaragoza, España \\ cfa@unizar.es
}

\begin{abstract}
Resumen
Se discute la regulación de los "datos abiertos" u "open data", tomando como ejemplo el contexto español. A dichos efectos en primer lugar se ocupa de recoger una aproximación a lo que se denomina "open data" o "datos abiertos". En segundo lugar presenta, sintéticamente, la regulación española que se ocupa de ellos. En tercer lugar expresa que la normativa vigente requiere que, en su puesta en acción por medio de las notas legales, los responsables de su redacción deben atender a criterios democráticos. Finalmente, se concluye proponiendo que los datos abiertos sean diseñados en conformidad a la normativa sobre reuso de datos abiertos, protección de datos personales, delimitación de responsabilidad de las prestaciones de servicios y la que da pautas para el ejercicio de servicios administrativos concretos.
\end{abstract}

Palabras clave: Datos abiertos. Regulación. Protección de datos personales. Metodología jurídica. España.

\section{Introducción}

Este artículo realiza una aproximación a la normativa que comienza a promulgarse con el fin de producir la explotación, uso e integración social de la puesta a disposición de los usuarios de Internet de ingentes informaciones, datos abiertos u "open data" sobre la realidad, aprovechando las virtualidades que la misma red ofrece. El caso ejemplar considerado es la regulación española. A estos efectos el trabajo se articula de la siguiente forma: En primer lugar se ocupa de recoger una aproximación a lo que se denomina "open data" o "datos abiertos". En segundo lugar presenta, sintéticamente, la regulación española que se ocupa de ellos. En tercer lugar expresa que la normativa vigente requiere que en su puesta en acción sea considerado otro tipo de regulación cuyo contenido se expone sucintamente. Finalmente se realiza una conclusión.

\section{2. ¿Qué son los "open data"?}

\subsection{Definiciones}

Se habla de datos abiertos pese a que no existen definiciones claras acerca de qué son. Aca-

\begin{abstract}
The regulation of the "open data" is discussed, taking as an example the process that happens in Spain. For these purposes, first, a discussion of the concept of "open data" is provided. Secondly, the Spanish regulation that deals with them is synthetically overviewed. Thirdly, it is also stated requires that current legislation must be designed according to democratic criteria. Finally, it is concluded that open data are designed in accordance with these juridical rules: the norms on open data reuse, the normative on personal data protection, the normative on the responsibility for the provision of services, and the juridical guidelines that exist for the specific administrative services.
\end{abstract}

Keywords: Open data. Juridical regulation. Personal data protection norms. Juridical methodology. Spain.

so, como vemos a continuación, lo más correcto sea expresar que es un "movimiento de opinión" dirigido a promover la oferta de información que está en Internet en régimen de libre uso.

La siguiente definición resume la filosofía del movimiento (Open definition, 2005-2011) (1):

A piece of data or content is open if anyone is free to use, reuse, and redistribute it - subject only, at most, to the requirement to attribute and/or sharealike.

También está argumentada como "idea" la definición de open data en la Wikipedia (2014), versión inglesa:

Open data is the idea that certain data should be freely available to everyone to use and republish as they wish, without restrictions from copyright, patents or other mechanisms of control.

Estas declaraciones muestran sus límites, y que no pueden superar el terreno de las ideas, intenciones o movimientos de opinión, ni, por lo tanto, convertirse en realidad. Esto es así porque preconizan algo imposible: el "libre acceso a los datos", al margen de los derechos existentes sobre ellos, una vez que los datos, como los programas de ordenador, no son libres: siempre 
están referidos a personas y a sus derechos. En otras palabras: forman parte de aplicaciones, servicios o programas. Es decir, en su uso, como en el de los programas de ordenador, hay que atender a los derechos de sus propietarios, sin poder ser éstos obviados como las definiciones presentadas proponen.

Esto es coherente, además, con la circunstancia de que en la primera de las definiciones mencionadas se excluye de la expresión datos, que se hace equivalente a conocimiento, a los programas de ordenador: "Software is excluded despite its obvious centrality because it is already adequately addressed by previous work" (Open definition, 2005-2011). Para la definición, por tanto, los datos o el conocimiento son de libre uso mientras que los programas no... Como indica esta excepción, la misma ha de tomarse como regla general, porque tanto los programas como los datos tienen fines y propietarios: han sido trabajados previamente por quienes los publican o se refieren a personas. Es decir, no existen, en puridad, los "datos abiertos" o de libre uso: siempre es preciso respetar los derechos de sus titulares, sean ellos empresas o personas físicas.

Pese a la contradicciones que presentan estas propuestas, lo cierto es que cada día es mayor la información que está accesible en Internet. Esto no tanto porque las empresas en las que se integran los técnicos que constituyen el movimiento "open data" lo hagan, una vez que por lo general sus datos no son de libre uso, y, si lo son ello, es o bien durante un breve periodo de tiempo destinado a la captación de posibles usuarios de los programas que los utilizan, o bien porque forman parte de un todo con el que las empresas persiguen que la publicidad adjunta les reporte beneficios. Otra cosa sucede, en cambio, con las informaciones o datos que proceden de individuos o de las administraciones como vemos a continuación.

SI existen datos abiertos ello es, especialmente, o bien porque los publican los mismos usuarios de la red -es el caso de las comunicaciones hechas a través de redes sociales como Twitter o los Blogs-, o bien porque, en lo referido a información pública, los Gobiernos la están colocando en forma respetuosa (más o menos) con la legislación vigente, haciéndola accesible a todos aquellos que los quieren utilizar. Esto último se ha visto incrementado por la expansión de la aceptación del principio político de transparencia de las actividades de las Administraciones Públicas, prescrito, por ejemplo, por la Ley española 19/2013 de 9 de diciembre, y la obligación consiguiente que las mismas tienen de dar acceso de la información que almacenan y tratan en su vida diaria, en forma respetuosa con el carácter y contenido de la información publicitada.

En la expansión de la idea de datos abiertos influye también la existencia de programas en funcionamiento que permiten la recuperación en forma rápida y eficaz de la información que está accesible en Internet. Si nos fijamos en el buscador Google, por ejemplo, éste cada vez más proporciona respuestas más atinadas a aquellos usuarios de los que aprende, contando con su aceptación, cuáles son sus perfiles en forma de hábitos, usos y gustos. Virtualidades que proporcionan a la empresa ingresos por la explotación del acceso a los datos de las empresas que los publican y tratan (2).

\subsection{Servicios}

¿Cuál es la razón de la existencia de expresiones de interés e incluso movimientos a favor de los "open data"? Como vamos a ver es una razón económica: ilustra al respecto la reseña de algunas aplicaciones o servicios que los utilizan o que pueden utilizarlos, lo que hacemos, brevemente, a continuación.

Así, por ejemplo, cabe desarrollar servicios que detecten, en función de cuál sea la temperatura interior y exterior de una vivienda, el momento en el que la calefacción o la refrigeración deban ser apagadas automáticamente. La temperatura interior se averigua por la información sobre el ambiente recogida por termómetro, la exterior por otro termómetro $y$, si se quiere programar el servicio para un periodo de tiempo determinado, por las previsiones hechas por un servicio meteorológico. Lo mismo ocurre con la iluminación de casas y vías públicas o calles. Así se entiende que sería deseable tecnológicamente, por ello, que los datos que permiten realizar las predicciones y las nuevas aplicaciones fueran abiertos o de libre uso.

Se pueden desarrollar, igualmente, servicios que provean por medio del uso de semáforos y otras señales de tráfico o sensores adecuados, gestionados por instituciones públicas, rutas abiertas para el movimiento de servicios de urgencia o el transporte de vehículos que hacen la distribución de mercancía. Sería deseable que los datos o la información que permiten crear estas rutas estuvieran abiertos para poderse desarrollar nuevos sistemas.

Existen/existirán programas de ordenador capaces de indicar si hay o no plazas libres en un aparcamiento de automóviles a la vez que hacen previsiones de ocupación para el futuro, permitiendo reservarlas para un determinado 
momento. Los datos en este caso pueden ser proporcionados por instituciones públicas o por empresas que gestionan los aparcamientos.

Estos ejemplos dan cuenta de que efectivamente se están construyendo servicios o programas, contando con datos, que pueden auxiliar a los ciudadanos. La construcción de estos servicios o programas cuenta con la información captada de la naturaleza y la vida diaria por sensores; y también la de los ciudadanos anonimizada, capturada, acumulada y tratada atendiendo a pautas de comportamiento individuales, de usuarios de los servicios, que se ponen a disposición de quien la ha generado o a la de otros usuarios/ciudadanos que los adquieran. En todo caso —de ello nos ocupamos a continuación-, no son datos abiertos como propugnan los movimientos de opinión sobre "open data" porque siempre existe algún derecho sobre ellos.

\section{La regulación sobre uso de datos abiertos}

Hemos dicho que una importante fuente de información "abierta" está constituida por los datos de carácter público, que cada vez más son accesibles en formatos estándar en Internet, siendo puestos a disposición de ciudadanos o empresas por las Administraciones a través de los correspondientes reglamentos. Es por ello que la regulación sobre datos abiertos está referida a datos de carácter público. Así lo permiten y regulan las directivas europeas y leyes estatales referidas a reutilización de datos públicos, acceso a la información y transparencia. Sumariamente la regulación vigente es la siguiente.

- Regulación española: Ley 37/2007, de 16 de noviembre, sobre el reuso de información del sector público; y Real Decreto 1495/2011, de 24 de octubre, que desarrolla la ley 37/2007, y Ley19/2013, del 13 de diciembre sobre Transparencia, Acceso a la información pública y Buen Gobierno.

- Regulación europea: El 13 de abril de 2013, el Consejo de la Unión Europea revisó la Directiva 2003/98/EC on the Reuse of Public Sector Information; y el 13 de junio de 2013 el Parlamento europeo adoptó una resolución legislativa "on the proposal for a directive of the European Parliament and of the Council amending Directive 2003/98/EC on re-use of public sector information (COM(2011)0877C7-0502/2011-2011/0430(COD)).

En esta normativa se regula, como sus enunciados dicen, la forma de "reutilizar" por servicios, aplicaciones, sistemas o programas concretos los datos que reciben en el ejercicio de sus funciones las Administraciones mediante su publicación en Internet, respetando siempre las garantías que se da en su recopilación a la preservación de la propiedad intelectual e industrial y a la protección de datos personales. También se prescribe que cada información suministrada por las correspondientes Administraciones de ámbito estatal, autonómico y local, establezca las notas o avisos legales referidos al uso de dicha información (art. 7 Real Decreto 1495/ 2011).

Los avisos legales establecen en muchas ocasiones la no obligación de las Administraciones en relación a la continuación de la publicación de datos abiertos, circunstancia que ha de ser tenida en cuenta por los proveedores de servicios que utilizan los datos públicos a efectos de que establezcan los oportunos acuerdos con las Administraciones con el fin de garantizar la continuación de la publicación de los mismos y la subsistencia de la oferta de sus servicios.

Con lo hasta aquí señalado observamos que la puesta en acción de la regulación sobre datos abiertos apela mediante la prescripción de la elaboración de notas o avisos legales por quienes suministran la información, a establecer una guía para el acceso a los datos. Es decir hay que acudir a la regulación existente sobre programas y servicios mediante los cuales se "atesoraron" inicialmente los datos y contrapesar, expresándolo en forma de avisos legales, los distintos valores a los que la información reutilizada responde. De ello nos ocupamos en el siguiente apartado.

\section{Los avisos legales}

A lo anterior se añade, por tanto, la consideración de que la normativa a la que también hay que atender en el diseño de servicios a partir de datos abiertos, es la existente para regular el funcionamiento de los servicios propios de la sociedad de las tecnologías de la información y la comunicación desde hace ya cierto tiempo (los años setenta del siglo XX): protección de datos personales, medidas de seguridad, firma electrónica, acceso electrónico de los ciudadanos a los servicios públicos, preservación de la propiedad intelectual e industrial y las medidas generales del ordenamiento jurídico previstas para la preservación y atribución de responsabilidad. En este apartado nos referimos a algunas de estas exigencias básicas.

\subsection{La elaboración de propuestas jurídicas} atendiendo a principios democráticos

No quedan suficientemente satisfechos los principios jurídicos cuando la propia Unión Europea, 
al establecer y actualizar Directivas sobre reutilización de la información de las Administraciones Públicas, se limita a expandir las posibilidades de crecimiento que tiene la construcción de "servicios/programas", en cuanto se considera que este mismo desarrollo es capaz de crear riqueza o empleo. Sin duda que esta es "una" obligación de su función, pero ahí no acaban "todas" sus obligaciones para con los ciudadanos. Nos referimos a que las instituciones públicas en el Estado de Derecho han de ocuparse de promover todo tipo de actividades democráticas, es decir la realización de nuevos diseños o programas, en nuestro caso, en razón a garantizar con ello el cumplimiento de todos los fines del Estado de Derecho, como a continuación exponemos, y no sólo el relativo a la generación de empleo.

Esto es así porque ha de recordarse que un sistema político democrático, y las instituciones que lo integran, no sólo está justificado por la creación de empleo, sino que lo está porque (Carbonell, 2008) atiende (a) a la garantía e impulso de los mecanismos y principios jurídicos fundamentales, reconocidos en las constituciones y hechos realidad en la vida diaria de los países donde las mismas funcionan; (b) a la satisfacción de un requisito previo para el ejercicio de mecanismos y principios —el acceso a información-; y (c) a su puesta en acción mediante la gobernanza en cuanto ella es una de las principales políticas o filosofías que cabe seguir para la puesta en realidad de los principios democráticos y el diseño de servicios/programas, una vez que los principios de gobernanza/prudencia han de respetarse en la construcción y puesta en funcionamiento de servicios automáticos, porque éstos no pueden atender por si mismos a resolver toda la complejidad que sucede en la realidad en el campo de aplicación de ese tipo de servicios. La gobernanza está definida por el Diccionario como

Arte o manera de gobernar que se propone como objetivo el logro de un desarrollo económico, social e institucional duradero, promoviendo un sano equilibrio entre el Estado, la sociedad civil y el mercado de la economía.

Lo anterior implica reconocer la expansión en el ámbito público, como prácticas o usos propios de los gobernantes (incluyendo en la expresión a todos los funcionarios públicos que ponen en acción a los tres poderes), de los principios, técnicas o usos de gobierno propios del ámbito empresarial. Esto es lo mismo que decir: la puesta en acción de la eficiencia y las reglas del mercado como criterio de acción preferente de los poderes públicos.
Este estilo de acción o política no impide lo ya expresado, es decir asumir que el respectivo poder ha de ser ejercido, al establecer las notas legales para el uso de los datos abiertos, en forma compatible con la puesta en práctica de los principios propios del Estado de Derecho, que resumen la acción de la democracia. La democracia es el valor en forma de estructura de funcionamiento que, por mandato legal, gobierna la acción de los poderes públicos; es decir, todos aquellos asuntos sobre los que éstos son competentes según el ordenamiento propio de los Estados de Derecho en cuanto son agentes activos en la vida social y política de la sociedad del conocimiento (3).

Es conveniente recordar, en forma resumida, a estos efectos, el mensaje básico enviado por algunos de los estudiosos, filósofos del Derecho, a prácticos del Derecho proponiéndoles un modo de acción complejo para el ambiente reseñado desde un tiempo ya lejano como el constituido por los comienzos del siglo XX (Robles 2010), cuya complejidad es bueno recordar para el caso que nos ocupa.

Desde aquella época, justamente desde el comienzo de la obligación de los jueces de poner en ejecución el Código Civil alemán bajo su responsabilidad ante todos los casos que los ciudadanos les plantearan, surgieron consideraciones críticas con respecto a la idea de que la aplicación del Derecho estaba reducida a la realización automática de la subsunción del caso concreto en la Ley, como planteaban y presumían los Códigos liberales. Ehrlich, junto a los tratadistas y jueces que se integraron en el Movimiento de Derecho Libre, puso de manifiesto que el proceso de aplicación del Derecho no podía estar reducido a la subsunción una vez que la irremediable existencia de lagunas jurídicas hace que la mayor parte de las resoluciones judiciales sean creaciones "libres", de los mismos jueces, a efectos de no incurrir en la responsabilidad correspondiente por no tomar decisiones en casos, sometidos a su decisión por imperativo legal, cuyos supuestos y soluciones no coincidieran con los previstos por la Ley (Ehrlich, 1903). A partir de estas consideraciones surgieron a lo largo del siglo $X X$ hasta la actualidad múltiples reflexiones dirigidas a completar el proceso de aplicación del Derecho con otras explicaciones.

Algunas de las soluciones propuestas fueron las siguientes: el conocimiento de las concepciones y convicciones sociales (propuesta hecha por Ehrlich a través de la Sociología: el derecho vivo), la consideración de que el razonamiento jurídico está integrados por tópicos o lugares comunes que auxilian a la aplicación (Viehweg 
1974); el establecimiento de sistemas normativos auxiliares a la aplicación elaborados mediante el uso de la lógica contando con la construcción de la pirámide normativa que amplía racionalmente el ámbito legal (Kelsen 1979); la propuesta del estudio de las leyes atendiendo a que se interpretan a partir de la "precomprensión" de su contenido (Esser 1961; Engisch 1968; Gadamer 1977); el estudio de la aplicación judicial del Derecho atendiendo al amplio ámbito y contenido de las argumentaciones que en la misma se produce (Perelman 1979 y Alexy 1992); la consideración del acuerdo de legitimación social -el consenso-, al que están dirigidas las leyes y la organización estatal en su totalidad (los tres poderes) en las sociedades democráticas (Habermas 1993); la consideración de que todas las actividades humanas son realizadas atendiendo a un conocimiento de la realidad producido en el contacto mantenido con la misma realidad - "autopoiéticamente" (Maturana, 1988) - y no por la mera elucubración o desarrollo intelectual de las propuestas científicas...

Estas y otras propuestas estaban ocupadas, resumiendo, en poner énfasis en el contexto propio de las actividades jurídicas, a efectos de explicarlas y darles soluciones más complejas que las que establece la subsunción o el discurrir formal sobre los textos jurídicos. De forma similar, la gobernanza obliga a revisar la confianza en el automatismo de los servicios producidos a partir del reuso de los datos abiertos, y proponer en avisos legales que esos programas/servicios/sistemas/artefactos se construyan en su totalidad en forma respetuosa con las normas y principios jurídicos conforme a los cuales se recopilaron los datos administrativos y que quedan descritos sintéticamente en los siguientes apartados.

\subsection{La protección de datos personales}

El primer requisito a satisfacer por los servicios/programas es el del cumplimiento de la legislación sobre protección de datos personales. Esto es así porque, como hemos expresado, los sistemas/servicios se ponen en funcionamiento a partir de la utilización de los datos personales de sus usuarios. Debemos recordar que la regulación sobre sistemas de seguridad de la información es parte de la legislación sobre protección de datos, lo que ocurre, al menos, en España (4).

El primer dato es la existencia del consentimiento de una persona concreta para que se consideren sus datos personales como entrada/inicio de la aplicación. Los usuarios han de dar permi- so expreso, suficientemente documentado, al almacenamiento de dichos datos en la correspondiente base de datos personales.

La entidad responsable del servicio debe hacer constar la existencia de esta base de datos y los fines de la aplicación, programa o sistema, que hace uso de los datos personales en el correspondiente Registro de la Autoridad de protección de datos personales.

La recopilación, almacenamiento y uso de información y datos realizados a través de sensores también han de ser consentidos por los propietarios, poseedores o usuarios del lugar, vehículo e imagen cuyos elementos o características hayan sido "sensados" en cuanto que esta información es fácilmente identificable con la de la persona propietaria, poseedora o usuaria de la cosa concreta.

4.3. La garantía de responsabilidad sobre las propuestas de los servicios a los usuarios

Los proveedores de datos libres tienen que tener en consideración los problemas de responsabilidad que pueden surgir cuando se hace un uso efectivo de los servicios. Por ejemplo, para el caso de un accidente de tráfico: una colisión, ocurrida cuando alguien atiende a las indicaciones del sistema relativas a ocupar una plaza de aparcamiento reservada al usuario y resulta que la plaza no está libre sino ocupada. Estas posibilidades han de ser contempladas en un contrato a realizar entre el proveedor de los datos, el del servicio y la empresa que gestiona un aparcamiento o entre el mismo proveedor y el usuario.

Este aspecto es muy relevante cuando se utiliza información procedente de Administraciones Públicas: es frecuente que éstas declaren que están exoneradas de responsabilidad para el caso de uso de la información que ellas suministren. Así ocurre, por ejemplo, con la Agencia Española de Meteorología (s. a.) que en su aviso legal indica (5):

AEMET declina toda responsabilidad sobre los daños y perjuicios que pudieran ser ocasionados por la interpretación y el uso de la información puesta a disposición de los ciudadanos en este Web.

Todo lo anterior expresa que los proveedores de servicios elaborados con datos abiertos deben hacer unas claras previsiones sobre responsabilidades del uso de sus servicios y recogerlas en los contratos a realizar con quienes dan los datos y con quienes utilizan sus servicios (Ossowski, 2013). 
4.4. El cumplimiento de los requisitos administrativos en el caso de provisión de servicios legales

Una cuestión jurídica diferente a las anteriores es la referida a la provisión de servicios administrativos a ciudadanos. Nos referimos en este caso, por ejemplo, a la propuesta por el sistema de la cumplimentación de una solicitud para reclamar un derecho a la Administración; por ejemplo, el de escolarización de un niño en un colegio público o concertado. El sistema bien podría recopilar la documentación administrativa precisa referida al niño y a los padres (como certificados de nacimiento, declaración de renta, lugar de vivienda, lugar de trabajo...); y luego remitirla, junto a la solicitud correspondiente, a la instancia administrativa encargada de resolver conflictos para el caso de que existan más solicitantes que plazas de estudio.

En casos como el expresado, la relevancia de la atención a los aspectos jurídicos es añadida a los casos anteriores una vez que la cuestión de hecho está expresada detalladamente en normas y reglamentos, con lo cual ha de atenerse estrictamente a este contenido, claramente interpretable, en el momento de construirse el programa a efectos de clarificar responsabilidades (5). Por otro lado este tipo de servicios en los que se trata de acceder electrónicamente a las Administraciones Públicas requiere que los servicios se ajusten a las reglas y estándares establecidos por la ley referida justamente al Acceso electrónico a las Administraciones Públicas por parte de los ciudadanos (España, 2007).

\section{Conclusión}

El presente artículo ha presentado, atendiendo a experiencias concretas en desarrollo en materia de datos abiertos de carácter público, algunas de las razones por las que los servicios que utilizan datos abiertos han de ser diseñados desde un primer momento en conformidad con la legislación vigente en relación a la reutilización de "open data", pero también a materias como protección de datos personales, delimitación de responsabilidad de las prestaciones de los servicios y a la atención a un funcionamiento acorde con la legislación que especifique el ejercicio concreto de servicios jurídicos.

\section{Notas}

(1) Esta página web ilustra sobre el contenido y filosofía del movimiento protagonizado por los diseñadores de Internet.

(2) Ver algunas novedades sobre el desarrollo de algoritmos de búsqueda de Google en, por ejemplo Singhal (2013).
(3) Se hacen propuestas a este respecto en relación al ámbito local en Estados Unidos en Katz (2010).

(4) La Ley Orgánica 15/1999, de 13 de diciembre sobre la protección de datos personales se completó con el Real Decreto de 1720/2007, de 21 de diciembre, que aprobó el Reglamento que implementaba la ley Orgánica 15/1999. Este Reglamento también actualizó el Reglamento sobre medidas de seguridad para los archivos automatizados que contenían datos personales de 11 de junio de 1999.

(5) Ver un interesante estado del arte sobre esta cuestión en Casella (2011).

\section{Referencias}

Agencia Española de Metereología (s. a.). Nota legal. http://www.aemet.es/es/nota_legal (2014-03-31).

Alexy, Robert (1992) Begriff und Geltung des Rechts. Freiburg: Verlag Karl Alber, 1992. 201-206.

Carbonell, Miguel (2008), Constitution's functionality and social rights: outline of some problems.//Estudios Constitucionales, 6:2 (2008) 43-71.

Casella, Nuria (2011), Legal Ontology Engineering. Methodologies, Modelling Trends, and he Ontology of Professional Judicial Knowledge, Dordrecht: Springer 2011.

Ehrlich, Eugen (1903), Freie Rechtsfindung und freie Rechtswissenschaft, Leipzig; C.L. Hirschfeld,1903, 7-17.

Engisch, Karl, (1968) La idea de concreción en el derecho y en la ciencia jurídica actuales. Pamplona: EUNSA, 1968, 349-394.

España (2007). Ley 11/2007 de 22 de junio para el acceso electrónico de los ciudadanos a los servicios públicos.

Esser, Josep, (1961) Principio y norma en la elaboración jurisprudencial del derecho privado. Barcelona: Bosch, 1961, 309-339.

Gadamer, Hans George, (1977) Verdad y método: fundamentos de una hermeneútica filosófica. Salamanca: Sigueme, 1977, 360.

Habermas, Jürgen, (1993) Faktizität und Geltung. Beiträge zur Diskurstheorie des Rechts und der demokratischen Rechtsstaats , Frankfurt: Suhrkamp (1993) 208-237.

Katz, Ellen.D. (2010) Engineering the Endgame// Michigan Law Review.109:3 (2010) 349-386.

Kelsen, Hans (1979) Teoría general del Estado, México: Editora Nacional, 1979, 21-27.

Maturana, Humberto (1988), El árbol del conocimiento: las bases biológicas del conocimiento humano, Santiago de Chile: Editorial Universitaria, 1988, 159-165.

Open definition (2005-2011). http:// http://opendefinition.org (2014-03-23)

Ossowski, Sascha (2013). Agreement Technologies, Dordrecht: Springer 2013.

Perelman, Chaim (1979), La lógica jurídica y la nueva retórica. Madrid: Civitas, 1979, 176-233.

Robles, Gregorio (2010). Teoría del Derecho. Madrid: Civitas 2010.

Singhal, Amit (2013). Fifteen years on, and we're just getting started. // Google. Inside Search. 26-9-2013. http://inside search.blogspot.com.es/2013/09/fifteen-years-onand-were -just-getting.html\#uds-search-results (2014-03-30).

Viehweg, Theodor (1974) Topik und Jurisprudenz, München: Beck, 1974, 111-119.

Wikipedia contributors (2014). Open data. // Wikipedia. http://en.wikipedia.org/wiki/Open_data (2014-03-23).

Enviado: 2014-04-03. Aceptado: 2014-07-10. 